\title{
Critical discussion of light scattering and microscopy techniques for CNC particle sizing
}

\author{
Carole Fraschini, Grégory Chauve, Jean-François Le Berre, Steven Ellis, Myriam Méthot, Brian O'Connor and Jean \\ Bouchard
}

\begin{abstract}
KEYWORDS: Cellulose Nanocrystals, Particle Size, Length, Distribution Profile, Microscopy, Light Scattering, Electron Microscopy, Hydrodynamic Radius, Radius of Gyration
\end{abstract}

\begin{abstract}
SUMMARY: The determination of particle length and aspect ratio of acicular cellulose nanocrystals (CNCs) becomes crucial when CNCs are used as a catalyst or as a reinforcement additive or when their size is used as a quality control parameter for production. We measured particle dimensions and particle size distribution of CNCs using several experimental techniques based on electron and optical microscopy as well as static and dynamic light scattering. Electron microscopy can easily reveal the real dimensions (i.e. length and cross-section) of nonsymmetrical particles like CNCs while the lightscattering derived techniques cannot. With the latter, it is however possible to approximate the real dimensions using the appropriate theory. The different aspects of light scattering techniques and types of data that can be obtained are presented and detailed. For each technique used, measurements done with $\mathrm{CNCs}$ are compared to those obtained with spherical standard latex particles. The accuracy of the final data was also related to the time necessary to obtain it. This "accuracy over time" ratio is of importance when specific applications such as on-line quality control are targeted.
\end{abstract}

\section{ADDRESS OF THE AUTHORS:}

FPInnovations, 570 boulevard Saint-Jean, Pointe-Claire H9R 3J9 (Québec), Canada

Corresponding author: Carole Fraschini

(carole.fraschini@fpinnovations.ca)

\section{Introduction to Nanomaterials and Cellulose Nanocrystals} (CNCs)

The International Organization for Standardization has defined a solid nanomaterial as a particle with at least one dimension smaller than $100 \mathrm{~nm}$ (ISO/TS27687 2008). The widespread interest in nanoscience has arisen from the altered or enhanced properties of materials as the particle size decreases towards the atomic scale. These changes in material properties are due to a larger surface and the occurrence of quantum effects. Whereas a larger surface area promotes higher chemical reactivity as well as altered strength and electrical properties, quantum effects affect the electrical, magnetic and optical properties of materials. Aspect ratio and particle length of rod shaped nanoparticles also give rise to many interesting phenomena such as anisotropy and percolation in composites. These properties can be exploited to make newer and more effective products such as photonic crystals or nanocomposites with greatly improved mechanical properties.

Cellulose nanocrystals (CNCs) represent the crystalline region of individual cellulose microfibrils, held together by amorphous regions. Due to their low density, high aspect ratio and excellent mechanical properties, CNCs have the potential to replace, at least in part, toxic or nonbiodegradable materials mostly in the composite industry. The main challenge to increase usage of $\mathrm{CNCs}$ is to improve its compatibility with hydrophobic matrices by altering its surface chemistry.

CNCs are extracted from naturally occurring cellulose sources such as wood pulp, cotton, bacteria, and tunicates. Using strong acid hydrolysis conditions, the amorphous cellulosic parts of the cellulose microfibrils are dissolved thus releasing nanocrystals in the aqueous medium. Even with similar extraction processes, the variety of cellulose sources leads to CNCs of different average length and cross-sectional dimensions. The use of sulfuric acid imparts negatively charged acidic sulfate ester groups located at the surface of the crystals. These charges cause electrostatic repulsion between the CNCs and confer a robust stability to the resulting aqueous CNC suspensions (Dong et al. 1996; Revol et al. 1992). On the contrary, hydrochloric acid treatment does not introduce stabilizing negative charges onto the CNCs' surface, resulting in an unstable colloidal system. A subsequent chemical process is then required to make the CNCs stable in suspension (Araki et al. 2001; Azzam et al. 2010).

\section{Requirements for Particle Size Measurements of Nanomaterials}

Quality Control. Large scale production of CNCs became a reality when CelluForce ${ }^{\mathrm{TM}}$ Inc. opened its demonstration plant located in Windsor (Province of Québec, Canada, 2012). A precise measure of the particle size is clearly mandatory to guarantee a consistent production of high-quality CNCs. At the moment, no standard methods for size determination of polydisperse nanomaterials of acicular form, such as CNCs, exists.

Toxicity. Recently, there have been societal concerns about the possible adverse effects of manufactured nanomaterials. Nanomaterial properties differ from those of the parent bulk materials because of their smaller size and larger surface area, resulting in a greater reactivity and potentially different biological effects. Because such particles can have a negative effect on biological systems and ecosystems, their toxicological risks must then be ascertained and an accurate description of the product in terms of dimensions, chemistry and toxicity is now required by the authorities. Extensive evaluation of CNCs using standard ecotoxicological and mammalian test protocols has been done and has, to date, been shown to be practically non-toxic in each of the individual tests undertaken (Kovacs et al. 2010). CNCs have also recently obtained regulatory approval under Canada's New Substances Notification Regulations (NSNR) for 
unrestricted use in Canada and will shortly be added to Canada's domestic substance list.

$\boldsymbol{R} \& \boldsymbol{D}$ and Applications. To unleash the full potential of the outstanding properties of nanomaterials, the particles have to be as well dispersed as possible, especially in nanocomposites applications. Any alteration of this ideal individualization state will lead to nanoparticle flocculation, agglomeration or, in the worst case scenario, irreversible aggregation. CNCs are not always handled as a liquid suspension, but can be obtained in their solid form. CNCs form a hornified solid microstructure upon drying which may be difficult to further redisperse, even in water. The redispersion issue is often worse when the dispersing matrix is composed of non-polar solvents or molten polymers (Heux et al. 2000). Redispersion can be made possible only if the particles are prevented from strongly interacting with each other during the drying process. Redispersion in water was proven feasible by neutralization of the acidic sulfate ester groups on the CNCs surface prior to drying (Beck et al. 2012).

\section{Challenges of CNC Particle Size Measurements}

First of all, it is important to explain some of the reasons why the particle size of CNCs in suspension can vary. As mentioned, the stability of the colloidal aqueous suspension is due to the electrostatic repulsion forces created by the negatively charged sulfate ester groups located at the surface of the crystals. Parameters like CNC concentration, ionic strength, temperature or presence of an additional compound or impurities can easily disrupt this equilibrium, leading to agglomeration.

In the case of CNCs, particle size is a good indicator of the quality of the dispersion but the direct observation of individual nanoparticles remains a challenge and high resolution imaging or light scattering techniques are required.

\section{A Variety of Particle Sizing Techniques}

There are many instruments commonly used for the determination of particle size distribution (PSD) and particle dimension(s), each technique detecting size through its own physical principle. Numerous techniques based on microscopy, light interactions, electrical properties, sedimentation, sorting and classification exemplify the possibility to get access to the size of the particles. The accuracy of the technique is often related to the nanoparticle shape and its physicochemical features such as chemical composition, heterogeneity, topography, surface charge density, dispersing medium, viscosity, etc. (Fedotov et al. 2011). However, many techniques such as light scattering make the useful and convenient assumption that every particle is a sphere and the reported size is typically expressed as an equivalent spherical radius (or diameter). This approach is simplistic and of course not perfectly accurate especially in the case of acicular particles, such as fibers, rod-like particles (like CNCs) or needles, for which problems can arise. The only techniques that can describe particle size in all dimensions are electron microscopy and atomic force microscopy (AFM) which provide sufficient nanometer scale resolution capabilities for CNC imaging. However, the shape of CNCs may appear different depending on the technique used. For instance, in the case of AFM, tip- broadening effects make the assessment of whether the morphology observed is individual particles or several particles agglomerated side-by-side difficult.

Usually, particle size measurement techniques provide different but complementary information on the dimensions of particles. These measurements allow us to access either average particle size data or in some cases a distribution profile which gives an idea of the polydispersity within the sample (ISO1321 1996). Moreover, in the case of CNCs, the issue is two-fold due to the intrinsic asymmetry of the rod-like particle and their polydispersity in length as well as in width. Consequently, the comparison of particle size data obtained from different techniques is very tricky to establish.

In this report, the particle dimensions as well as the particle size distribution of spherical and rod-shaped particles (latex standards and CNCs, respectively) were investigated using light and electron microscopy as well as static and dynamic light scattering techniques. The basics of the different techniques are detailed and an attempt to transform equivalent spherical size data into real size is presented. For each technique, relevance of experimental data is considered taking into account the time necessary to get the final result.

\section{Materials and Methods}

\section{CNC Suspension Preparation and Characterization}

Aqueous CNC suspensions of 2 to $6 \%(\mathrm{w} / \mathrm{w})$ were prepared in the FPInnovations pilot plant in PointeClaire, Quebec, Canada from bleached softwood kraft pulp according to a procedure adapted from the literature (Dong et al. 1998). The final $\mathrm{pH}$ of the $\mathrm{CNC}$ suspensions ranged from 2.5 to 3 . The sulfur content $(0.85 \% \pm 0.05 \%$ $\mathrm{S}$ w/w) was measured by inductively coupled plasma atomic emission spectroscopy (ICP-AES).

\section{Latex Nanoparticles Suspension}

50 and $100 \mathrm{~nm}$ radius latex nanoparticle aqueous suspensions were used as standard spherical nanoparticles (Duke Scientific Corp., CA, USA).

\section{Field Emission Scanning Electron Microscopy}

The imaging was carried out with a Field Emission GunScanning Electron Microscope (Hitachi SU-70 FE-SEM, Japan) equipped with a scanning transmission electron detector. The CNC suspension was first dialyzed against deionized water then diluted to a concentration of about $0.01 \% \mathrm{w} / \mathrm{w}$ in water. $10 \mathrm{ml}$ of the suspension of interest was sonicated $\left(100 \mathrm{~J}_{\mathrm{ml}} \mathrm{m}^{-1}\right)$ followed by a negative staining procedure with uranyl acetate to enhance the contrast. One drop of the suspension was deposited onto a piece of parafilm. A special grid with a positively charged surface (Nanoplus, Dune Sciences, OR, USA) was placed in contact upside down with the deposited drop for 45 seconds. In the same manner, the wet grid was then placed directly in contact with a drop of $2 \%$ uranyl acetate solution. After 2 minutes, the excess liquid was immediately absorbed with a blotter paper. The grid was air-dried for at least one hour. Grid with casted dyed specimen was mounted on the microscope sample holder for imaging. At least twenty micrographs were recorded 
Table 1 - Parameters for CNC particle size measurements.

\begin{tabular}{|c|c|c|c|c|c|c|}
\hline & Sample volume & $\begin{array}{l}\text { Conc. min. } \\
\text { (w/w) }\end{array}$ & $\begin{array}{l}\text { Conc. max. } \\
(\mathrm{w} / \mathrm{w})\end{array}$ & $\begin{array}{l}\text { Total time } \\
\text { (h) }\end{array}$ & Raw data & PSDa \\
\hline FE-SEM & $100 \mu \mathrm{l}$ & $0.001 \%$ & $0.01 \%$ & 48 & Absolute length & yes \\
\hline DLS & $1 \mathrm{ml}$ & $0.05 \%$ & $0.05 \%$ & 0.5 & Average $r_{h}$ (as esv $\left.{ }^{b}\right)$ & yes \\
\hline NTA & $5 \mathrm{ml}$ & $0.00001 \%$ & $0.01 \%$ & 1 & Average $r_{h}\left(a s e^{e s v^{b}}\right)$ & yes \\
\hline SLS & $20 \mathrm{ml}$ & $0.00001 \%$ & $0.01 \%$ & 1 & Average $r_{g}$ (as esv $\left.{ }^{b}\right)$ & no \\
\hline
\end{tabular}

a particle size distribution

b equivalent spherical volume

at a magnification of $\times 70.000$ using an accelerating voltage of $10 \mathrm{keV}$ or above. The resolution of the microscope is around $2 \mathrm{~nm}$ under these conditions.

The determination of the $\mathrm{CNCs}$ length distribution profile was done using Image Pro analysis software. Because no automated analysis software is yet available for counting CNCs, we visually/manually counted single particles. Aggregates were discarded and only the well individualized CNC particles were measured and added to the inventory. It is highly recommended that at least 1,000 objects be counted to get representative distribution profiles and that no more than $25 \%$ of objects within a single image are discarded.

For standard particles, a latex suspension drop was deposited on a positively charged grid, which was then air-dried for at least one hour. The diameter of a hundred particles was measured. The magnification used was around 50,000x and the accelerating voltage was of $10 \mathrm{KeV}$.

\section{Dynamic Light Scattering (DLS)}

Particle size and particle size distributions were obtained using a Zetasizer Nano ZS (Malvern, UK) at $25^{\circ} \mathrm{C}$. This equipment allows the size measurement of particles ranging from one nanometer up to ten microns. A He-Ne laser light source $(633 \mathrm{~nm})$ was used to illuminate a representative dilute suspension of interest (CNCs and latex). It uses non-invasive backscatter detection at an angle of $173^{\circ}$ which improves the sensitivity, reduces multiple scattering issues and minimizes the effects of large particles contaminants like dust. The CNC suspension was diluted to $0.05 \%$ w/w with a $\mathrm{NaCl}$ solution (the final $\mathrm{NaCl}$ concentration being $5 \mathrm{mM}$ ). The ISO procedure (ISO1321 1996) recommends a concentration of $10 \mathrm{mM}$ but we chose a lower concentration to prevent any gelation or agglomeration issue. Samples were then filtered through a $0.7 \mu \mathrm{m} \mathrm{GF} / \mathrm{F}$ glass fiber filter in order to remove any of the biggest particles (dust, unreacted pulp, aggregates, etc.). A polystyrene (or quartz) cuvette was filled with $1 \mathrm{ml}$ of the filtered suspension. Each sample (latex or CNCs) was analyzed in triplicate. Because this technique assimilates any particle to spherical particles of equivalent volume, the CNCs measurement result is expressed as a hydrodynamic radius. To calculate the hydrodynamic radius of the particles, the theoretical viscosity and the refractive index of the continuous phase have to be known.

\section{Laser Light Scattering Microscopy}

The recently commercialized nanoparticle tracking analysis (NTA) microscope generates particle size profiles through videos of populations of nanoparticles in suspension moving under Brownian motion when illuminated by a laser light source (Nanosight Ltd, UK). A microscope equipped with a green laser $(532 \mathrm{~nm})$ allows the visualization of nano-scaled particles in real time and dynamically analyzes the particle paths over a suitable period of time. Despite the rapidity with which the particles move, such motion can be readily tracked using a CCD camera. The displacement of each tracked particle is associated to its translational diffusion coefficient $\left(D_{t}\right)$ from which the hydrodynamic radius can be retrieved. The NTA microscope detects particles at very dilute concentrations ranging from $0.00001 \%$ to $0.01 \% \mathrm{w} / \mathrm{w}$. Acquisition time is a function of the concentration and the polydispersity of the sample. Like DLS, no information on the sample is required to estimate the hydrodynamic radius of the particles, only the viscosity and the refractive index of the continuous phase must be provided.

\section{Static Light Scattering (SLS)}

The DAWN HELEOS II consists in a 18-angle static light scattering detector (MALS, Wyatt Technology Corporation, CA, USA). A well collimated, single frequency polarized laser light beam $(658 \mathrm{~nm})$ is used to illuminate a sample containing a suspension of the particles of interest. Intensity and angular dependence of the scattered light are then measured. MALS can measure particle sizes ranging from $10 \mathrm{~nm}$ up to $500 \mathrm{~nm}$. The signal intensity carries information about the molar mass, while the angular dependence carries information about the averaged size of the macromolecule, known as the root mean square (rms) radius, or radius of gyration $\left(r_{g}\right)$. Batch measurements can be done by either injecting a sample aliquot directly into the flow cell, or by introducing the sample in a container such as a scintillation vial. $r_{g}$ can be determined without knowing the sample concentration or the differential index of refraction $(d n / d c)$.

The most important parameters for the determination of CNC particle size, according to each of the abovedescribed technique, are tabulated in Table 1.

\section{Basics of Particle Size Measurements}

$\mathrm{CNC}$ particle size measurements are very challenging because of their size polydispersity (length and crosssection) as well as their acicular shape. Moreover, the measurements obtained with various techniques do not give access to the same data. We believe it is pertinent here to summarize the concepts behind each technique. 


\section{Electron Microscopy and Image Analysis Basics}

Traditionally in the pulp and paper industry, samples examined by scanning electron microscopy (SEM) are pulp fibers, surfaces and/or cross-sections of paper or board. Examination of materials such as CNCs deposited on grids is usually performed with a transmission electron microscope (TEM) but scanning transmission electron microscopy (STEM), which combines a transmission electron detector mounted onto a scanning electron microscope, can also be used. Even though electron microscopy allows direct examination of the particles, it is not suitable for quality or production control. Sample preparation and data analysis are laborious and timeconsuming. Moreover, as a relatively low number of nanoparticles can be examined at once, there is a real danger of misrepresentative sampling. In the United States, the National Bureau of Standards (NBS) recommends that a minimum of 10,000 images (not particles!) must be examined for statistical validity (Dragoo et al. 1987).

\section{Dynamic Light Scattering Basics}

Dynamic Light Scattering (DLS) is often referred to as Photon Correlation Spectroscopy (PCS) or Quasi-Elastic Light Scattering (QELS). The Malvern Zetasizer Series is one of the world's most widely used instruments for nanoparticle/colloid size measurements.

DLS measures the random movement of particles (Brownian motion) suspended within a liquid medium. The larger the particle, the slower its Brownian motion. The velocity of a particle under Brownian motion is defined by its translational diffusion coefficient $\left(D_{t}\right)$, which in turn can be used to determine the hydrodynamic radius $\left(r_{h}\right)$ of the particles as derived from the StokesEinstein equation. For spherical particles, the equation reads $(E q 1)$ :

$$
\mathrm{r}_{\mathrm{h}}=\left(\mathrm{k}_{\mathrm{b}} \mathrm{T}\right) /\left(6 \pi \eta \mathrm{D}_{\mathrm{t}}\right)
$$

where $r_{h}$ is the hydrodynamic radius of the particle (in $\mathrm{m}$ ), $\mathrm{k}_{\mathrm{b}}$ is the Boltzmann constant (in $\mathrm{J} . \mathrm{K}^{-1}$ ), $\mathrm{T}$ is the absolute temperature (in $\mathrm{K}$ ), $\eta$ is the medium viscosity (in $\left.\mathrm{kg} \cdot \mathrm{m}^{-1} \cdot \mathrm{s}^{-1}\right), \mathrm{D}_{\mathrm{t}}$ is the translational diffusion coefficient (in $\left.\mathrm{m}^{2} . \mathrm{s}^{-1}\right)$. It is noteworthy that experimental parameters like temperature and viscosity values should be stable and accurately known.

For rod-like shaped particles, the equation of $D_{t}$ reads (Eq 2) (Broersma 1981; De Souza Lima et al. 2002):

$$
\begin{aligned}
& \mathrm{D}_{\mathrm{t}}=\left(\mathrm{k}_{\mathrm{b}} \mathrm{T} /(3 \pi \mathrm{L})\right)\left(\delta-0.5\left(\gamma_{\perp}+\gamma \|\right)\right. \\
& \text { with } \delta=\ln (2 \mathrm{~L} / \mathrm{d}) \\
& \gamma_{\perp}=-0.193+0.15 / \delta+8.1 / \delta^{2}-18 / \delta^{3}+9 / \delta^{4} \\
& \gamma_{\|}=0.807+0.15 / \delta+13.5 / \delta^{2}-37 / \delta^{3}+22 / \delta^{4}
\end{aligned}
$$

The hydrodynamic radius $r_{h}$, also called Stokes radius, or Stokes-Einstein radius, represents the radius of a hypothetical hard sphere having the same diffusion coefficient or viscosity as the particle under examination. Because $\mathrm{CNCs}$ in suspension are non-spherical and solvated, hypothetical hard spheres have no real existence and the hydrodynamic radius is only indicative of the 'apparent' size of the dynamic hydrated/solvated CNC particle (hence the terminology 'hydrodynamic'). In the case of rod-like particles like CNCs, a slight change in the length will directly affect the measured DLS size while changes in the cross-section will hardly affect the diffusion velocity. Tough useful and easily determined, $r_{h}$ represents the hydrodynamic size which can significantly differ from the true physical size.

The coefficient $D_{t}$ depends not only on the physical size and size-related behavior (e.g. diffusion or viscosity), but also on any surface structure and texture (topography, roughness, charges), as well as concentration and size of ions present in the medium. If the particle shape changes in a way that affects the diffusion velocity, then $r_{h}$ will also change. The presence of electrolytes also plays a role in the particle diffusion velocity by altering the electrostatic interaction through its electrical double layer referred to as the electroviscous drag (Schumacher, van de Ven 1987; 1991). Electrical double layer systematically appears at the surface of any object (solid/porous particle, gas bubble, liquid droplet, etc.) when immersed into a liquid and is mostly apparent in systems characterized with a large surface to volume ratio like colloids and nanoparticles. The addition of any electrolyte screens the surface charges of CNCs, reducing the electrostatic repulsion that prevents them from agglomerating and may consequently cause flocculation. Since CNCs do not undergo swelling or compaction upon the addition of electrolyte, the variation of the measured particle size is a pure artifact. Flocculation and agglomeration phenomena can thus affect the particle size measurement and the resulting effect depends on the amount and the size of ions introduced. For chitin crystallites suspensions, the addition of $1 \mathrm{mM} \mathrm{NaCl}$ compresses the electrical double layer from a thickness of 19.2 to $9.6 \mathrm{~nm}$ ( $\mathrm{Li}$ et al. 1996). The apparent diameter of the rod decreases from $46.4 \mathrm{~nm}$ to $27.2 \mathrm{~nm}$ while the axial ratio increases from 4.3 to $7.3 \mathrm{~nm}$.

In the ideal case of spherical particles, if the particles are small compared to the wavelength $\lambda$ of the laser used (i.e. particle size less than $\lambda / 10$ ) the light scattered by the particle will be mainly isotropic (equal in all directions) and the size can no longer be estimated accurately. The Rayleigh scattering intensity, which originates from the particle scattering, is inversely proportional to $\lambda^{4}$ meaning that to increase the intensity of the light scattered, a shorter wavelength should be used. The scattering intensity is also directly proportional to the

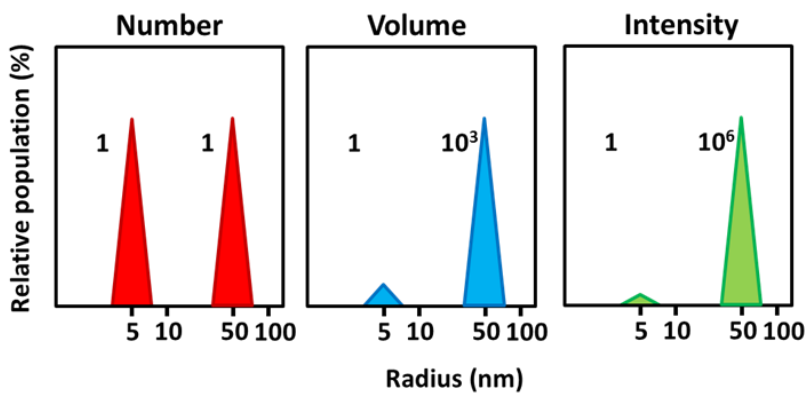

Fig 1 - Relative populations of particles of 5 and $50 \mathrm{~nm}$ radius in equal number expressed in number, volume and intensity as determined by light scattering. 


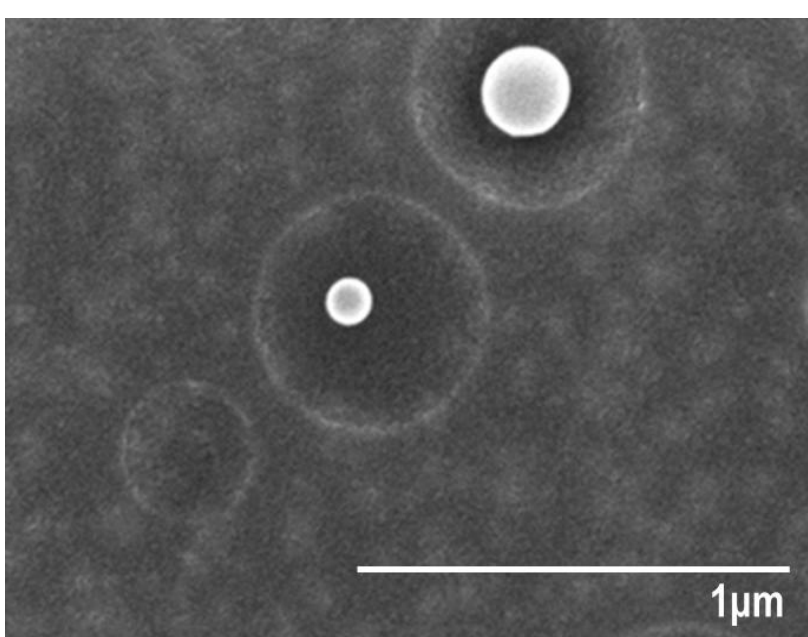

Fig 2 - FE-SEM micrograph of a mixture of spherical latex particles of 50 and $100 \mathrm{~nm}$ in radius.

sixth power of the size meaning that a $50 \mathrm{~nm}$ particle will scatter one million times as much light as a $5 \mathrm{~nm}$ particle. In a polydisperse sample (which is the case for CNC suspensions), the contribution of small particles to the total light scattered is negligible compared to the larger particles which will screen the light scattered from the smaller ones. For example, if two populations of particles of 5 and $50 \mathrm{~nm}$ radius in equal numbers are mixed together, the peak ratios expressed in relative number, volume and scattered light intensity distributions are $1: 1$, $1: 10^{3}$ and $1: 10^{6}$, respectively (Fig 1 ).

\section{Laser Light Scattering Microscopy Basics}

Nanoparticle Tracking Analysis (NTA), a technique commercialized in 2006, is able to determine sizes of particles ranging from 10 up to $1000 \mathrm{~nm}$, the lower detection limit being dependent on the refractive index of the nanoparticles.

It combines a laser light scattering microscope with a conventional charge coupled device (CCD) camera that allows visualization and real-time monitoring of particles moving under Brownian motion. Using the light scattered by particles, the NTA software identifies and tracks individual particles correlating their movement to their size according to the following equation $(E q 3)$ derived from Stokes-Einstein equation:

$$
\mathrm{r}_{\mathrm{h}}=\left(2 \mathrm{k}_{\mathrm{b}} \mathrm{T}\right) /\left(3 \pi \eta \overline{(x, y)^{2}}\right)
$$

where $r_{h}$ is the hydrodynamic radius (in $m$ ), $k_{b}$ is the Boltzmann constant (in $\mathrm{J}^{-1} \mathrm{~K}^{-1}$ ), $\mathrm{T}$ is the absolute temperature (in $\mathrm{K}$ ), $\eta$ is the medium viscosity (in $\mathrm{kg} \cdot \mathrm{m}^{-1} \cdot \mathrm{s}^{-}$ $\left.{ }^{1}\right),{\overline{(x, y)^{2}}}^{2}$ is the mean-squared speed of a particle moving under Brownian motion (in $\mathrm{m}^{2} \cdot \mathrm{s}^{-1}$ ).

Unlike DLS, the scattered light is only used to detect and track the particle in the video frame. Intense light scattered from large particles can mask the detection/tracking of small particles. A careful adjustment of the analytical settings (gain, shutter and detection threshold) as well as the sample concentration is essential to track all moving particles being captured by the video.

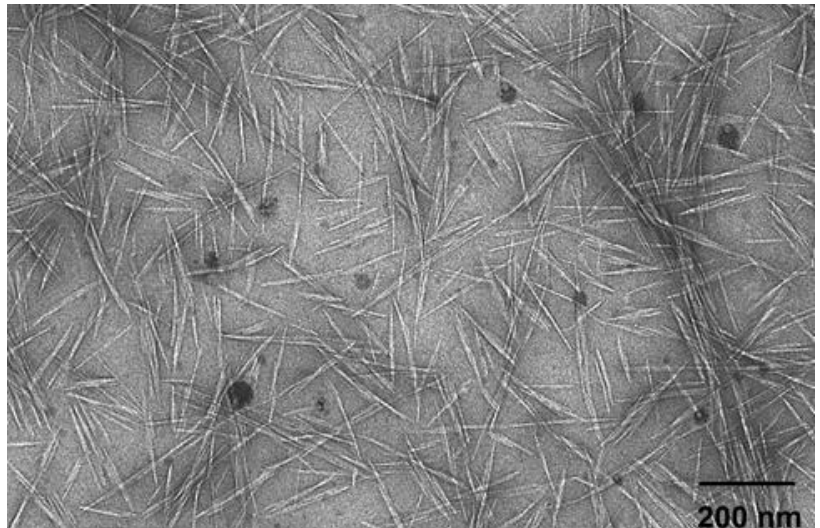

Fig 3 - FE-SEM micrograph of a CNC suspension.

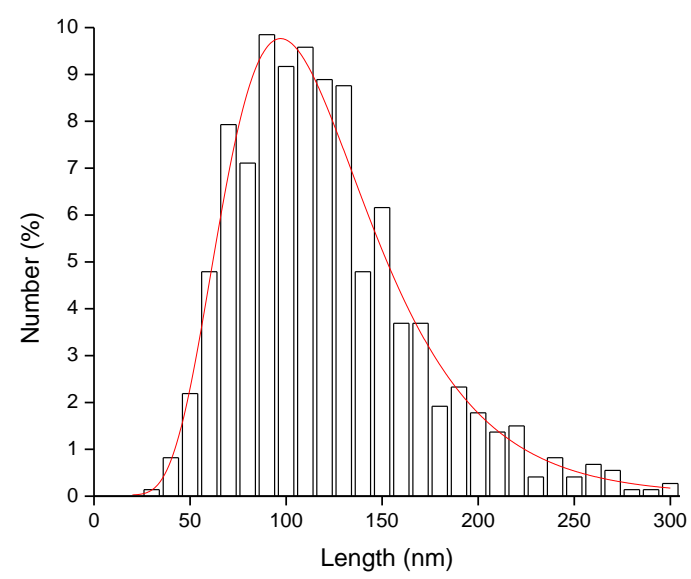

Fig 4 - Length distribution profile from electron micrographs of CNCs.

\section{Static Light Scattering Basics}

The radius of gyration $r_{g}$, or root mean square (rms) radius is proportional to the average distance of each point of a molecule relatively to its center of mass, weighted by the square of the distance from the center of mass $(E q 4)$. In simpler terms, $\mathrm{r}_{\mathrm{g}}$ is a measure of the size of the particle related to its mass distribution.

$$
\mathrm{r}_{\mathrm{g}}=\sqrt{ }\left\langle\mathrm{r}_{\mathrm{g}}{ }^{2}\right\rangle=\sqrt{ }\left(\left(\sum \mathrm{r}_{\mathrm{i}}{ }^{2} \mathrm{~m}_{\mathrm{i}}\right) /\left(\sum \mathrm{m}_{\mathrm{i}}\right)\right)
$$

where $r_{g}$ is the radius of gyration, $r_{i}$ is the distance from the mass center to the atom $i$ of the molecule (in $m$ ), $m_{i}$ is the mass of the atom $i$ (in $g$ ).

$r_{g}$ can be used to retrieve the 'conventional' dimensions of a molecule if its shape is known :

- for a uniform dense sphere with a radius a: $r_{\mathrm{g}}=\mathrm{a} \sqrt{ }(3 / 5)$

- for a hollow sphere with a radius $a: r_{g}=a$

- for a random coil polymer with an averaged end-toend length $L: r_{g}=L \sqrt{ }(1 / 6)$

- for a rigid rod with length $\mathrm{L}: \mathrm{r}_{\mathrm{g}}=\mathrm{L} \sqrt{ }(1 / 12)$

Size particle measurements carried out using light scattering techniques lead to averaged radius values, and thus cannot reveal the heterogeneity of the particle in terms of size and geometry, especially in the case of acicular particles like CNCs. To ov ercome this issue, mathematical equations can be used to estimate the real size, as it will be described later in this report. 


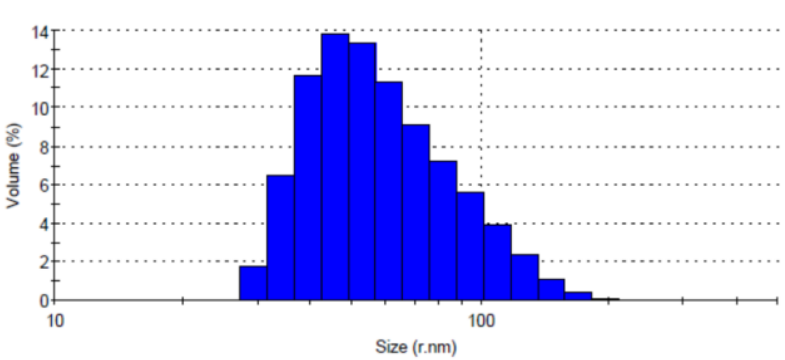

Fig 5 - DLS histogram of $50 \mathrm{~nm}$ latex suspension in $5 \mathrm{mM} \mathrm{NaCl}$.

\section{Results and Discussion}

\section{Electron Microscopy}

As depicted in Fig 2, electron microscopy provides an accurate estimation of the radius for a mixture of $50 \mathrm{~nm}$ and $100 \mathrm{~nm}$ standard latex particles. These results strictly conform to the specification sheets provided by the supplier.

The dimensions of CNCs were measured from the electron micrographs (Fig 3) according to the method described in the experimental section. Typically, CNCs exhibit lengths ranging from 20 to $300 \mathrm{~nm}$. For all samples, the negative staining reveals that most particles are composed of multiple crystallites presumably linked by amorphous cellulose. The lengths of 1,230 individual particles were counted and the corresponding histogram is shown in Fig 4.

The asymmetrical distribution was successfully fitted using a log-normal function. The number-average length $\mathrm{L}_{\mathrm{n}}$ and the standard deviation calculated from the size distribution are $116 \pm 48 \mathrm{~nm}$ whereas the weight-average length $\mathrm{L}_{\mathrm{w}}$ is equal to $136 \mathrm{~nm}$, giving a polydispersity index $\left(\mathrm{PDI}=\mathrm{L}_{\mathrm{w}} / \mathrm{L}_{\mathrm{n}}\right)$ of 1.17 .

Though the resolution of the electron microscope is not high enough to get an accurate particle width, we have estimated a cross-sectional width of $\sim 9 \mathrm{~nm}$. This value is considerably higher than the values usually found in the literature for similar substrates $(\sim 5 \mathrm{~nm})$. This discrepancy can be explained by the slightly milder hydrolysis conditions compared to those usually used. A lower sulfuric acid concentration combined with a higher temperature of reaction provides a higher yield which is consistent with higher particle size (unpublished work).

Electron microscopy imaging can reveal any shape of a material: spherical, acicular, ill-defined, etc., but it remains a time-consuming method as it requires the sample to be prepared, dried and imaged. After imaging, every image has to be manually processed making particle size determination not a straightforward method.

\section{Dynamic Light Scattering (DLS)}

Fig 5 shows a DLS histogram of $50 \mathrm{~nm}$ latex particles in $5 \mathrm{mM} \mathrm{NaCl}$. The hydrodynamic radius is found to be 49.2 $\pm 13.7 \mathrm{~nm}$.

Fig 6 presents a DLS histogram of $\mathrm{CNCs}$ in the presence of $5 \mathrm{mM} \mathrm{NaCl}$. The distribution profile is represented by a log-normal function and the hydrodynamic radius is $21.9 \pm 4.5 \mathrm{~nm}$.

At this point it is critical to examine the theory behind the calculations in order to have a better understanding of these readings.

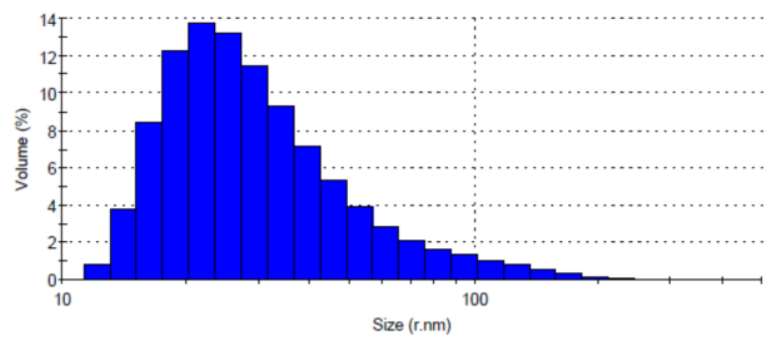

Fig 6 - DLS histogram of CNC suspension in $5 \mathrm{mM} \mathrm{NaCl}$.

\section{Dimensions Derived from Hydrodynamic Radius $\mathrm{r}_{\mathrm{h}}$}

As mentioned in the Introduction section, the diffusion velocity of particles moving in a fluid depends on the physical size and size-related behavior (e.g. viscosity). The particles in movement experience frictional resistance and their velocity is related to this frictional resistance by frictional coefficients. These coefficients are a function of the viscosity of the medium in which the particles are immersed. Most experiments are carried out in water or in dilute aqueous buffers/electrolyte solutions. The viscosity $(\eta)$ of water strongly decreases with increasing temperature. Experimental results are commonly adjusted to $20^{\circ} \mathrm{C}$, the temperature at which the viscosity of water is $0.001002 \mathrm{~kg} \cdot \mathrm{m}^{-1} \cdot \mathrm{s}^{-1}$. Values of $\eta$ for water at other temperatures are tabulated in the CRC Handbook of Chemistry and Physics (CRC Handbook of Chemistry and Physics 1998).

The translational $\left(f_{t}\right)$ and rotational $\left(f_{r}\right)$ frictional coefficients are expressed by the following equations $(E q$ 5 and $E q$ 6):

$$
\begin{aligned}
& \mathrm{f}_{\mathrm{t}(\text { sphere })}=6 \pi \eta \mathrm{r} \\
& \mathrm{f}_{\mathrm{r}(\text { sphere })}=8 \pi \eta \mathrm{r}^{3}
\end{aligned}
$$

where $\mathrm{r}$ is the radius of the equivalent sphere.

$E q 5$ and $E q 6$ show that translational friction ( $1^{\text {st }}$ power of radius) is less sensitive to particle size than rotational friction $\left(3^{\text {rd }}\right.$ power of radius). However, since light scattering techniques measure only $D_{t}$ coefficients, this paper will focus specifically on translational friction coefficients. Equations for rotational diffusion coefficients can be found in appropriate references (Tirado, Garca de la Torre 1979; Tirado, Garca de la Torre 1980; Garca de la Torre, Bloomfield 1981).

Non-spherical particles exhibit more complex properties than simple spheres. The translational frictional coefficient of a spheroid equals $(E q 7)$ :

$\mathrm{f}_{\mathrm{t}(\text { spheroid })}=\mathrm{f}_{\mathrm{t}(\text { sphere })} \cdot \mathrm{f}_{\mathrm{P}}$

where $f_{t(\text { sphere) }}$ represents the translational friction coefficient of a sphere of equivalent volume (StokesEinstein's law) and $f_{P}$ is the Perrin translational friction factor. If the particle is assumed to be a sphere having a radius $\mathrm{r}$ and a volume $\mathrm{V}=(4 / 3) \pi \mathrm{r}^{3}, \mathrm{f}_{\mathrm{t}}$ and $\mathrm{f}_{\mathrm{p}}$ are defined as follows ( $E q 8$ and $E q 9)$ :

$$
\begin{aligned}
& \mathrm{f}_{\mathrm{t}(\text { sphere })}=6 \pi \eta \mathrm{r}=6 \pi \eta(3 \mathrm{~V} / 4 \pi)^{1 / 3} \\
& \mathrm{f}_{\mathrm{P}}=\left(2 \mathrm{p}^{2 / 3}\right) / \mathrm{S}
\end{aligned}
$$

where $\mathrm{p}$ is the axial ratio and $\mathrm{S}$ is the Perrin factor. 
Table 2 - Perrin factors (S) of spheres, ellipsoids and cylinders.

\begin{tabular}{|c|c|c|c|c|}
\hline Perrin factor & Sphere & Oblate ellipsoida & Prolate ellipsoida & Cylinderb \\
\hline S & 2 & $(2$ atan $) / \xi$ & 2atanh $\zeta / \xi$ & $\sqrt[3]{12(\ln p+\gamma)}$ \\
\hline
\end{tabular}

In the case of spheroids, the axis about which rotation occurs is called the symmetry axis and its half-length is $a$. The half-lengths of the two other axes are equal to $b$. The axial ratio is defined as $\mathrm{p}=a b$. Oblate ellipsoids which are pancake-shaped show $\mathrm{p}<1$ and prolate ellipsoids which are cigar-shaped show p $>1$ (see Fig 7).The latter are often used as models for rod-shaped/cylindrical particles (like $\mathrm{CNCs}$ ) and give quite reasonable results if ( $\mathrm{Eq} \mathrm{10)}$ :

$$
\mathrm{b}_{\text {ellipsoid }}=\sqrt{ }(3 / 2) \mathrm{b}_{\text {cylinder }} \text { and } 2 \mathrm{a}=\mathrm{L}_{\text {cylinder }}
$$

where $\mathrm{L}$ is the length of the cylinder.

As cylinders do not exhibit narrowing ends like prolate ellipsoids, their frictional resistance is somewhat different. Moreover, the translational frictional coefficients are different depending on whether the long axis (half-length $a$ ) occurs in parallel or perpendicular to the direction of movement. However, particle sizers systematically measure averaged value of translational diffusion coefficient over all orientations. For spheres, all semi-axes are equal, so $\mathrm{p}=1$.The Perrin factors $\mathrm{S}$ for spheres, ellipsoids and cylinders are listed in Table 2.

Fig 7 shows two particles having the same axial ratio p>1 but different shapes (i.e. prolate ellipsoid and cylinder).

If we consider CNC particles having dimensions of 116 $\mathrm{nm}$ long and $9 \mathrm{~nm}$ wide (axial ratio $\mathrm{p}=12.9$ ) as measured by electron microscopy, the Stokes-Einstein equation $(E q$ 1) combined to Perrin factors $\mathrm{S}$ (Table 2) leads to a hydrodynamic radius of 17.8 and $19.9 \mathrm{~nm}$ for prolate ellipsoid-shaped particles and cylinder-shaped particles, respectively. The average radius value of $21.9 \mathrm{~nm}$ obtained by DLS is in quite good agreement with the theoretical calculations. However, it is crucial to keep in mind that theoretical values of hydrodynamic radii do not take into account the electroviscous drag brought by the presence of the electrical double layer, which may increase the expected size of the particles under examination. It is also important to note that only averaged values are obtained from the above-listed calculations and then cannot reveal the complexity of the heterogeneous CNC material, which exhibits a broad length distribution ranging from 20 to $300 \mathrm{~nm}$.

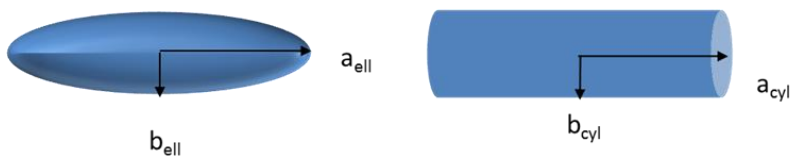

Fig 7 - Schematic representation of a prolate ellipsoid-shape (left) and cylinder-shaped particles (right) having the same axial ratio (if $a_{e l l}=a_{c y l}$ and $b_{e l l}=b_{c y l}$ ).

\section{Nanosight ${ }^{\mathrm{TM}}$ Microscopy (NTA)}

For $50 \mathrm{~nm}$ latex particles, the size measurement is accurate, giving a value of $r_{h}$ of $49 \pm 11 \mathrm{~nm}$ which conforms to the instrument specifications.

Fig 8 shows the CNC distribution profile obtained using the Nanosight ${ }^{\mathrm{TM}}$ microscope. The hydrodynamic radius is equal to $37 \pm 29 \mathrm{~nm}$. There is a discrepancy with the results obtained from conventional dynamic light scattering. In DLS measurements, electrolytes are usually used to collapse the electrical double layer but as they cause interferences in the nanoparticles detection and tracking, the NTA experiments were carried out without any additional electrolyte. The electrical double layer may be thicker and could explain the overestimated hydrodynamic radius obtained.

\section{Static Light Scattering (SLS)}

For latex particles of $50 \mathrm{~nm}$ in radius, the experimental value found was $35.0 \pm 0.8 \mathrm{~nm}$ which is in good agreement with the estimated theoretical radius of gyration of $38.7 \mathrm{~nm}$ for a uniform and dense sphere $\left(\mathrm{r}_{\mathrm{g}}=\mathrm{a} \sqrt{ }(3 / 5)\right.$ with $\left.\mathrm{a}=50 \mathrm{~nm}\right)$.

Fig 9 shows the Zimm plot obtained for an aqueous CNC suspension. A Zimm plot is a graphical double extrapolation used to analyze most light-scattering data. A set of light scattering experiments consists in measuring $\mathrm{Kc} / \mathrm{R}_{\Theta}$ at various concentrations and various scattering angles which is then plotted against $\left(\sin ^{2}(\theta / 2)+\mathrm{kc}\right) . \theta$ is the scattering angle, $\mathrm{K}$ is an optical constant, $\mathrm{c}$ is the concentration, $\mathrm{R}_{\theta}$ is the Rayleigh ratio and $\mathrm{k}$ is an arbitrary constant chosen to provide a convenient spread of the data. Mathematical extrapolation of the experimental results to $\mathrm{c}=0$ and $\theta=$ 0 allows us to determine the radius of gyration using the slope and intercept (automatically calculated by the ASTRA 6.0 software). The averaged radius of gyration of a CNC suspension extracted from the Zimm plot is $88.7 \pm 5.3 \mathrm{~nm}$.

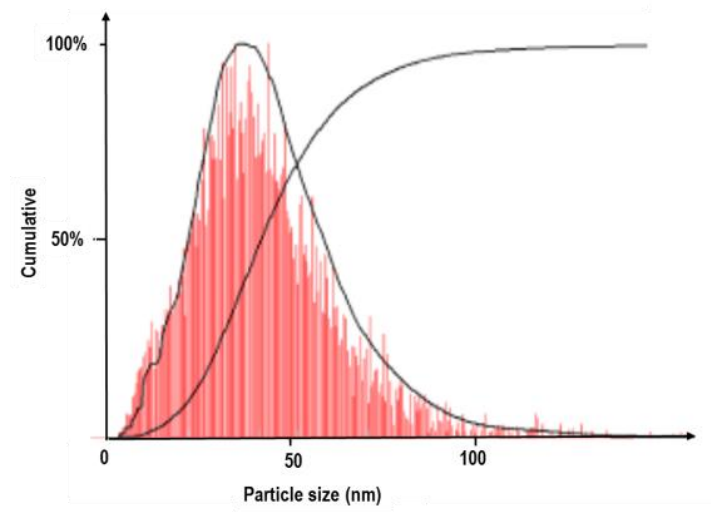

Fig 8 - NTA curve of a CNC suspension in water. 


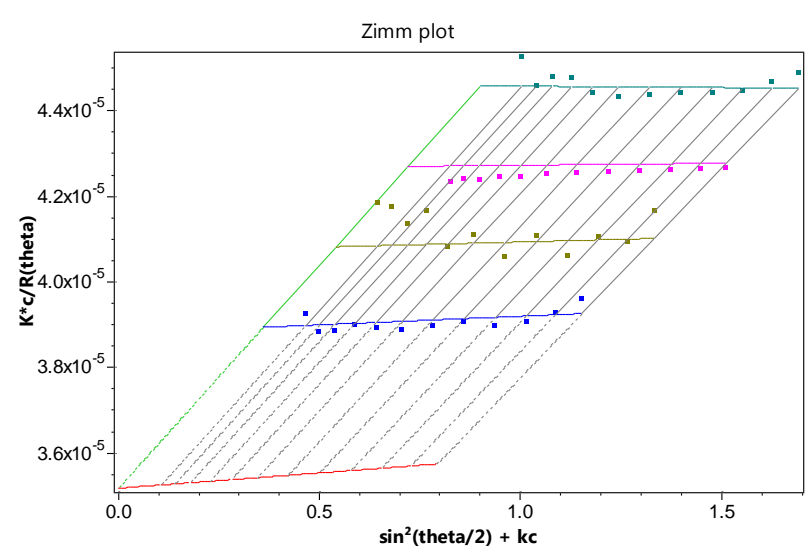

Fig 9 - Zimm plot of a CNC suspension in water.

\section{Dimensions Derived from Light Scattering Measurements}

As mentioned, it is possible to access information about the real size of an object when its geometry is known. For rod-shaped objects like CNCs, $r_{g}$ is a function of the length $\left(\mathrm{L}_{\text {rod }}\right)$ and the diameter of the cross-section $\left(\mathrm{D}_{\text {rod }}\right)$ as shown in $\mathrm{Eq}$ 11:

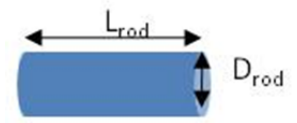

$\mathrm{r}_{\mathrm{g}}=\sqrt{ }\left(\mathrm{L}_{\text {rod }}^{2} / 12+\left(\mathrm{D}_{\text {rod }} / 2\right)^{2} / 2\right)$

Since $\mathrm{L}_{\text {rod }}{ }^{2} / 12 \gg\left(\mathrm{D}_{\text {rod }} / 2\right)^{2} / 2$, the term involving $\mathrm{D}_{\text {rod }}$ can be neglected, giving as a first approximation ( $E q$ 12):

$$
\mathrm{L}_{\mathrm{rod}}=\mathrm{r}_{\mathrm{g}} \sqrt{ } 12
$$

The experimental $r_{g}$ value of $88.7 \mathrm{~nm}$ corresponds to a calculated length of $307 \mathrm{~nm} \mathrm{(Eq} \mathrm{12).} \mathrm{Furthermore,}$ according to the light scattering theory, the contribution of small particles to the total light scattered is extremely small and the larger particles will screen the scattered light from the smaller ones. Consequently, the longest CNC particles which exhibit a length of about $300 \mathrm{~nm}$, even if there are just a few, will scatter more light than the smaller ones. This could explain the overestimated $r_{\mathrm{g}}$ determined experimentally.

For non-spherical particles, such as rod-shaped CNCs, it is necessary to access simultaneously $r_{g}$ and $r_{h}$ to provide the 'real' dimensions. As seen before, $r_{g}$ (from SLS measurement) can be used to retrieve the information about the length, $\mathrm{L}_{\text {rod }}$. Knowing the hydrodynamic radius (from DLS measurement) and the approximate electrical double layer thickness, it is possible to estimate the diameter of the cross-section of the rigid rod $D_{\text {rod }}$ using the hydrodynamic radius.

Table 3 - Summary of $50 \mathrm{~nm}$ radius standard spherical latex particle size measurements.

\begin{tabular}{lcc}
\hline & Data type & Experimental radius $(\mathrm{nm})$ \\
\hline FE-SEM & PSD & $50 \pm 1$ \\
DLS & $r_{h}$ & $49 \pm 14$ \\
NTA & $r_{h}$ & $49 \pm 11$ \\
SLS & $r_{g}$ & $35 \pm 1$ \\
\hline
\end{tabular}

The volume of rod-shaped particles is given by (Eq 13):

$$
\mathrm{V}=\pi \mathrm{L}_{\text {rod }}\left(\mathrm{D}_{\text {rod }} / 2\right)^{2}
$$

Its equivalent spherical volume is equal to $(E q 14)$ :

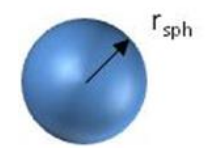

$\mathrm{V}=4 / 3 \pi \mathrm{r}_{\mathrm{sph}}^{3}$ with $\mathrm{r}_{\mathrm{sph}}=\mathrm{r}_{\mathrm{h}}-\mathrm{EDL}_{\text {thickness }}$

The combination of $(E q 11)$ to $(E q$ 14) gives (Eq 15):

$$
D_{\text {rod }}=\sqrt{ }\left(\left(16\left(r_{h}-e_{E D L}\right)^{3}\right) /\left(3 r_{g} \sqrt{ } 12\right)\right)=4 \sqrt{ }\left(\left(r_{h}-e_{E D L}\right)^{3 /} \quad\left(6 r_{g} \sqrt{ } 3\right)\right)
$$$$
\text { [15] }
$$

From experimental data obtained using static (SLS) and dynamic light scattering (DLS) and assuming an electrical double layer thickness of $2 \mathrm{~nm}$ (which corresponds to the difference between the theoretical and experimental radius of gyration obtained by DLS), the diameter of the cross-section of CNCs we calculated from ( $E q 15)$ is $11.7 \mathrm{~nm}$, which is in the same range as the value of $\sim 9 \mathrm{~nm}$ estimated from the electron microscopy images. These images also revealed that most of the particles are made of clustered parallel crystallites (usually 2 or 3 fragments). The difference in the crosssection measurements likely originates from these clusters (agglomerates or aggregates) that have been discarded from the electron microscopy measurements (averaging the particle size distribution towards the lower sizes) but not from the light scattering measurements.

\section{Conclusions}

Table 3 and 4 summarize the experimental data obtained using field emission scanning electron microscopy (FESEM), dynamic light scattering (DLS), nanoparticle tracking analysis (NTA) and static light scattering (SLS) for $50 \mathrm{~nm}$ radius standard spherical latex and CNC particles in aqueous suspensions.

Obviously, different techniques based on different measuring principles produce different results. For latex spherical particles, the experimental radius lies in the range 45-50 $\mathrm{nm}$ depending on the technique used. CNCs exhibit a mean particle length of $116 \mathrm{~nm}$ with a crosssection of $9 \mathrm{~nm}$, as determined by electron microscopy. When various light scattering techniques are combined together, the length is found to be $307 \mathrm{~nm}$ (from SLS data) and the cross-section $11.7 \mathrm{~nm}$ (from DLS and SLS data). But the question is to know which technique gives the most accurate result.

Table 4 - Summary of CNC particle size measurements.

\begin{tabular}{lccc}
\hline & Data type & $\begin{array}{c}\text { Experimental } \\
\text { Length }(\mathrm{nm})\end{array}$ & $\begin{array}{c}\text { Experimental } \\
\text { radius }(\mathrm{nm})\end{array}$ \\
\hline FE-SEM & $\mathrm{PSD}$ & $116 \pm 48$ & $\mathrm{n} / \mathrm{a}$ \\
DLS & $\mathrm{r}_{\mathrm{h}}$ & $\mathrm{n} / \mathrm{a}$ & $22 \pm 5$ \\
NTA & $\mathrm{r}_{\mathrm{h}}$ & $\mathrm{n} / \mathrm{a}$ & $37 \pm 29$ \\
SLS & $\mathrm{r}_{\mathrm{g}}$ & $307^{*}$ & $89 \pm 5$ \\
\hline
\end{tabular}




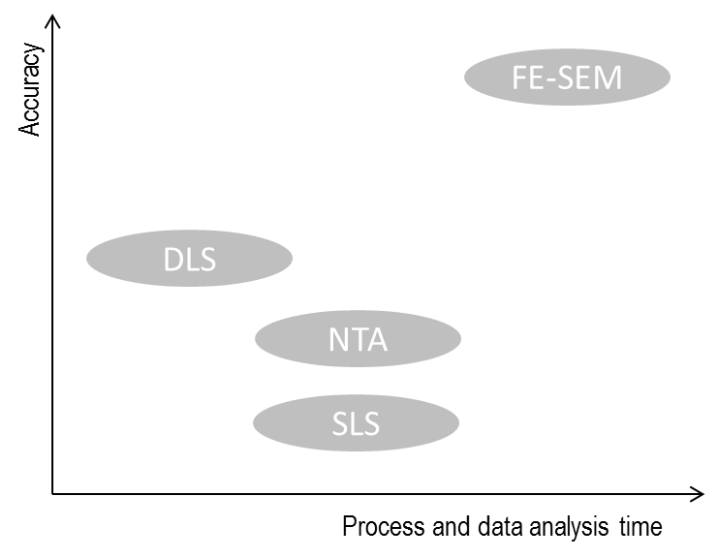

Fig 10 - Accuracy vs. time required to obtain the final result.

Many people think that 'seeing is believing' and for them, electron microscopy technique is considered to be the most suitable technique. However, this technique is not trouble-free and soft materials must be manipulated with extreme care because mishandling can distort the sample and hence alter final size determination. Moreover, this expensive technique is quite timeconsuming and subjective namely because a huge number of objects have to be counted to get a representative view of the whole sample. It is however quite easy to get reliable length histograms and real dimensions.

On the other hand, light scattering measurements can rapidly measure the size of particles in their native environment almost as an on-line measurement. The major issue in the case of colloids is the thickness of the electrical double layer which can significantly alter the "apparent" size of the particle. When no counterions are present, the particle will appear much thicker than the real particle. The addition of salt will help collapse the electrical double layer. However, in the presence of high ionic strength, the compression of the electrical double layer will promote agglomeration and bigger particles. A careful monitoring of key parameters that can affect the Brownian motion of the particles (i.e., ionic strength) is crucial to get reliable results. Representing an acicular particle by an equivalent spherical volume appears very hazardous at first glance and in the end is not an easy task. Length and width are the most descriptive values to describe CNCs. The real question is: how should we describe a non-spherical 3-D object with one unique number? Relevant theoretical equations have to be derived for true size evaluation. Polydispersity within samples is also an issue since most of these light scattering derived techniques cannot handle multimodal or non-normal/asymmetrical distributions. These techniques give only volume or mass-averaged values leading to overestimated size measurement results. With the light scattering techniques, the NTA system of the Nanosight ${ }^{\mathrm{TM}}$ microscope has the advantage to handle multimodal distributions. By doing so, it is possible to retrieve number-average distributions. Like DLS or SLS, only a modelized equivalent spherical radius can be obtained.

In an attempt to validate a reference technique that can be used for quality control purposes, it is legitimate to sort the techniques by taking into account the time required to prepare the sample, to process and analyze the data as a function of the accuracy of the measurement. This is particularly important when production quality control has to be monitored in real time under continuous flow. Fig 10 shows a schematic qualitative description of the accuracy of each technique versus the time required from sample preparation to final result.

NTA and DLS provide qualitative information that can be useful for quality control purposes. Combined, the latter to an SLS equipment could allow the user to retrieve both length and width only if the shape is known but if the real size distribution (length and width) is required, it can only be obtained by EM. Even if electron microscopy is the most accurate method, two days of analyzing time is not acceptable in a production unit, not to mention the prohibitive price of running and servicing such a piece of equipment.

\section{Acknowledgements}

This research was supported by the ArboraNano Network, the Transformative Technology Program of Natural Resources Canada and the Ministère des Ressources Naturelles et de la Faune du Québec.

\section{Literature}

Araki J, Wada M, Kuga S (2001): Steric stabilization of a cellulose microcrystal suspension by poly(ethylene glycol) grafting, Langmuir, 17(1), 21-27.

Azzam F, Heux L, Putaux JL, Jean B (2010): Preparation by grafting onto, characterization, and properties of thermally responsive polymer-decorated cellulose nanocrystals, Biomacromolecules, 11(12), 3652-3659.

Beck S, Bouchard J, Berry R (2012): Dispersibility in water of dried nanocrystalline cellulose, Biomacromolecules, 13(5), 1486-1494.

Broersma S (1981): Viscous force and torque constants for a cylinder, J Chem Phys, 74(12), 6989-6990.

CRC Handbook of Chemistry and Physics (1998): CRC Press Inc., Boca Raton, FL.

De Souza Lima MM, Wong JT, Paillet M, Borsali R, Pecora R (2002): Translational and Rotational Dynamics of Rodlike Cellulose Whiskers, Langmuir, 19(1), 24-29.

Dong XM, Kimura T, Revol JF, Gray DG (1996): Effects of ionic strength on the isotropic/chiral nematic phase transition of suspensions of cellulose crystallites, Langmuir, 12(8), 20762082.

Dong XM, Revol JF, Gray DG (1998): Effect of microcrystallite preparation conditions on the formation of colloid crystals of cellulose, Cellulose, 5(1), 19-32.

Dragoo AL, Robbins CR, Hsu SM (1987): A critical assessment of requirements for ceramic powder characterization, Adv Ceramics, 21, 711-720.

Fedotov P, Vanifatova N, Shkinev V, Spivakov B (2011): Fractionation and characterization of nano- and microparticles in liquid media, Anal Bioanal Chem, 400(6), 1787-1804.

Garca de la Torre J, Bloomfield VA (1981): Hydrodynamic properties of complex, rigid, biological macromolecules: Theory and applications, Q Rev Biophys, 14(1), 81-139. 
Heux L, Chauve G, Bonini C (2000): Nonflocculating and chiral-nematic self-ordering of cellulose microcrystals suspensions in nonpolar solvents, Langmuir, 16(21), 82108212.

ISO 1321 (1996): Particle size analysis - Photon correlation spectroscopy.

ISO Technical Specification ISO/TS 27687 (2008) Nanotechnologies - Terminology and definitions for nanoobjects - Nanoparticles, nanofibers and nanoplates. International Organization for Standardization.

Kovacs T, Naish V, O'Connor B, Blaise C, Gagné F, Hall L, Trudeau V, Martel P. (2010): An ecotoxicological characterization of nanocrystalline cellulose (NCC), Nanotoxicology, 4(3), 255-270.

Li J, Revol JF, Marchessault RH, Gray DG (1996): Rheological properties of aqeous suspensions of chitin crystallites, J Coll Interf Sci, 183, 365-373.
Revol JF, Bradford H, Giasson J, Marchessault RH, Gray DG (1992): Helicoidal self-ordering of cellulose microfibrils in aqueous suspensions, Int J Biol Macromol, 14(3), 170-172.

Schumacher GA, van de Ven TGM (1987): Brownian motion of charged colloidal particles surrounded by electric double layers, Faraday Discuss Chem Soc, 83, 75-85.

Schumacher GA, van de Ven TGM (1991): Brownian motion of rod-shaped colloidal particles surrounded by electric double layers, J Chem Soc Faraday Trans, 87(7), 971-976.

Tirado MM, Garca de la Torre J (1979): Translational friction coefficients of rigid, symmetric top macromolecules. Application to circular cylinders, J Chem Phys, 71(6), 2581-2588.

Tirado MM, Garca de la Torre J (1980): Rotational diffusion of rigid, symmetric top macromolecules. Application to circular cylinders, J Chem Phys, 73(4), 1986-1993.

Manuscript received November 5, 2013

Accepted January 8, 2014 